\title{
An Improved Algorithm for Image Compression Based on Biorthogonal Wavelet, DCT and Competitive Neuronal Networks
}

\author{
Amel Bey Boumezrag
}

\begin{abstract}
Current advances in research are the direction of new alternatives, combining different methods to communicate quickly and store a growing amount of data more efficiently: this mostly comprises the digital images which have invad our daily lives. That is why, this paper is devoted to the presentation of a digital compression algorithm based on improving the exploitation of the advantages of existing algorithms. It also aims to contribute to improving the performance of the transmission, and ensuring storage with a high compression ratio and low distortion.

In an initial stage, as pre-treatment, the image is decomposed into sub-bands (approximation sub-band + various details sub-bands), using a wavelet transform biorthogonal. Then, comes the phase of compression: Discrete Cosine Transform (DCT) is used to encode the approximation sub-band, which contains most of the information, followed by scalar quantization. In the last stage, the details of the sub-bands are encoded using vector quantization grouped into sub-bands using neural competitive learning.

I want to end this paper by demonstrating the effectiveness of this method, which is applied to compression of voluminous images, remote sensing, provided by the station of the National Meteorology Office in the visible, and infrared channels covering the area $\left(+47^{\circ} 19^{\circ}\right.$ North and South and West $16^{\circ}$ East $16^{\circ}$ ). For compression ratio values of over $97.2994 \%$, experimental results obtained show that these are faithfully reproduced, and these are not accompanied by artifact. Moreover, the storage memory has been significantly reduced.
\end{abstract}

Index Terms - Image compression, wavelet, DCT, competitive neuronal network, vector quantization, coding.

\section{INTRODUCTION}

Digital images play very important role in our daily lives. This has various domains of applications, such as mobile telecommunications, storage medical images, fingerprints, web and multimedia, TV transmissions, remote sensing images, telemedicine, video conferencing, etc. However, the volume of images generated has increased significantly. Hence, the step of compression has become extremely important in the minimization of storage space and transmission capacity. Many methods of image compression have been developed; some are applied with or without loss of information. There are traditional algorithms which carry out compression without loss, but are no longer sufficient to achieve performance in reducing volumes of information, such as: the RLE technique (Run Length Encoding),

Manuscript received July 26, 2015; revised January 15, 2016.

Amel Bey Boumezrag is with the Department of Electronic Engineering, Faculty of Science, University of Laghouat Algeria, BP 37G, Algeria (e-mail: am_by@yahoo.fr).
Huffman coding, and LZW (Lempel Ziv Welch).

New algorithms, which compress without loss, have been developed on the basis of powerful tools, such as: the wavelet transform, the discrete cosine transform (DCT), or the fractal approach [1]. Among the methods of high compresson are: JPEG (Joint Photographic Expert Group) [2], which is widely employed, JPEG2000 and [3]. They offer a good rate of compression.

However, more artifacts appear in them as tiles (blocks effect) and the execution time is very slow, especially in the case of large images. To reduce the distortions caused by the methods with high compression ratio, we have adopted a compression method based on the advantages of wavelet transform, the DCT and vector quantization, offering appropriate solutions for managing large volumes for a negligible loss of information, and high compression ratio. The principal steps and experimental results obtained are presented in the following sections.

\section{GENERAL DESCRIPTION OF THE COMPRESSION-DECOMPRESSION ALGORITHM}

The global process of compression-decompression of an image passes through a series of processing steps and the conversion of original information. In Fig. 1, we present the general process of compression-decompression that is adopted, the steps in this chain of compression/ decompression are as follows:

Step1: Pretreatment "decomposition into sub-bands" using discrete wavelets Transform (DCT);

Step2: DCT transform and scalar quantification of approximation sub-band;

Step3: Vector quantization of details sub-bands using neuronal competitive learning.

\section{A. Pretreatment "Decomposition into Sub-bands" Using $D C T$}

As the first step, the image to be compressed, is decomposed into a set of different resolution sub-images (sub-bands) using bi-orthogonal wavelet transform, corresponding to an approximation image (low-frequency coefficients), which contains the majority of the information inherent in the original image, and three sub-images contain the high frequency in accordance with horizontal, vertical and diagonal orientations.

\section{1) Discrete wavelet transform}

DWT based on image compression adopts the fast algorithm for two dimension. The original image decomposes into four sub parts after passing the image into 
high pass filter and low pass filter. The four sub-bands are the $L, H, V$ and $D$ respectively. $L$ is a low sub-band of the appeoximation image. $H$ is high frequency sub-band of the horizontal details of the image. $V$ is a high frequency sub-band of the vertical details of the image. $D$ is a high frequency sub-band of the diagonal details of the image [4]. This process is called the first level of wavelet decomposition. The low frequency sub-band can be continually decomposed into four sub-bands.

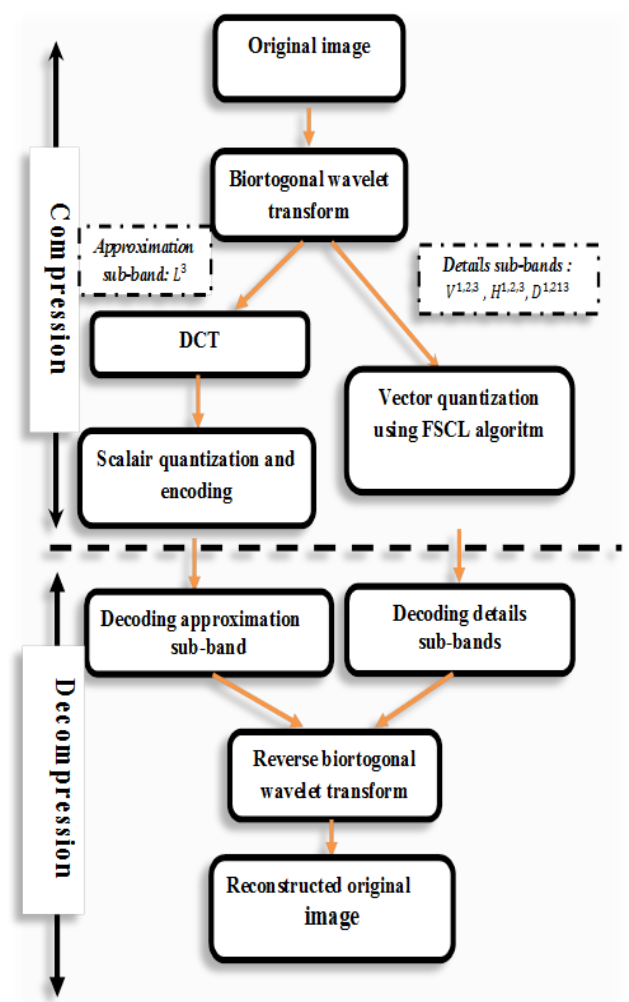

Fig. 1. Compression-Decompression process adopted.

Fig. 2 shows three level wavelet transform of an image test, where $L^{i}, V^{i}, H^{i}, D^{i}(i=1, \ldots, 3)$, are respectively, the low band, vertical, horizontal and diagonal sub-bands generated after three stage transformation. The various tests shown when we by choose a resolution 2-3, we obtain a reconstructed image of high quality. To determine the appropriate compression sub-bands, we conduct a statistical analysis of the wavelet coefficients characterizing each sub-band.

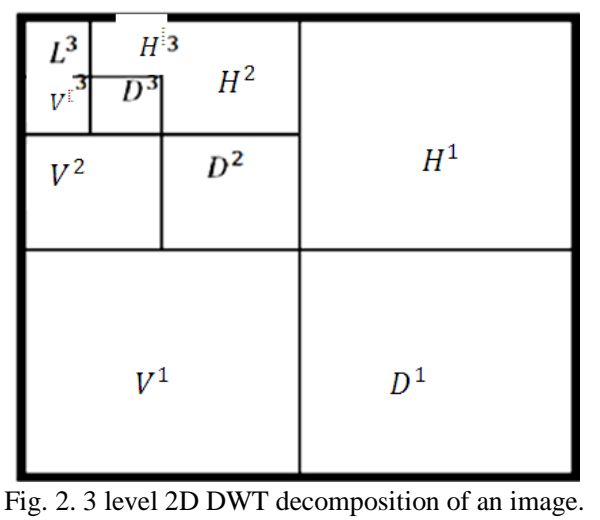

\section{2) Statistical analysis of wavelet sub-bands}

For each sub-band obtained after wavelet transform, the probability density function PDF (normalized histogram) was established. In Fig. 3, the probability densities of the coefficients of horizontal, vertical and diagonal details for one level decomposition are given.

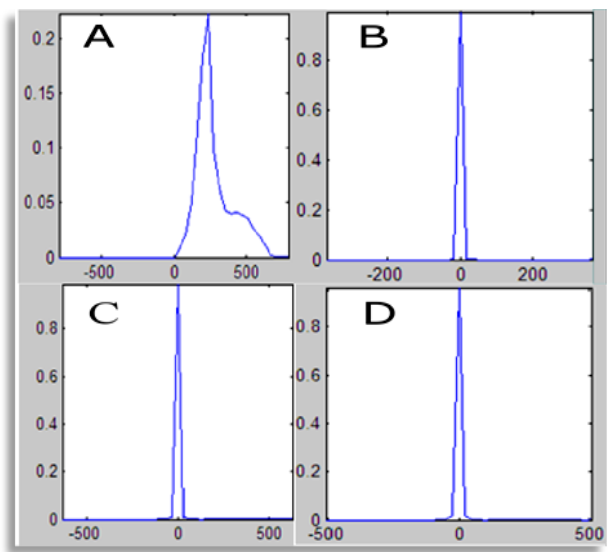

Fig. 3. (A): Low band wavelet coefficient for one level decomposition, (B): Vertical band distribution for one level, (C): Horizontal band distribution for one level, (D): Diagonal band distribution for one level decomposition.

We see that the wavelet coefficients globally follow the same evolution and they are for the greater part pointed (low variance), with a mean close to zero for all directions $(V, H$, $D)$ and all resolutions. This shows that in each sub-band there is a large number of wavelet coefficients of low value, providing insignificant information. The values of energy show a grater concentration of information in the lateral details that in the diagonals. In addition, the low band is characterized by a very high variance and maximum energy. It therefore, contains the major part of the original information.

Given all the properties described above, the low sub-band is encoded by a scalar quantization with minimum loss of information, and details sub-bands by vector quantization.

\section{B. DCT Transform and Scalar Quantification of Approximation Sub-band}

As shown earlier, the statistical and psychovisual proprieties of approximation sub-band (low sub-band) are the same as that of an original image. Consequently, the compression must be done with minimal losses. We tested a DCT based quantization method. That is used in many compression and transmission codec's, such as JPEG and MPEG [5], [6] (Fig. 4).

To do this, the low sub-band was divided into adjacent blocks of $8 \times 8$ pixels and the discrete cosine transform (DCT) is calculated for each block. We obtained a matrix whose elements are the coefficients of the DCT transform, containing two types of coefficients: DC and AC. The DC coefficient, represents the average of the pixels belonging to the current block (first element of the transformed matrix), the remaining elements are AC coefficients.

The discrete cosine transform of a block $f(m, n)$ formed by $N \times M$ pixels is defined by the following equation:

$$
F(u, v)=\frac{4 c(u)_{c}(v)}{N^{2}} \sum_{m=0}^{N-1} \sum_{n=0}^{N-1} \cos \left(\frac{(2 m+1)_{\pi u}}{2 N}\right) \cos \left(\frac{(2 n+1)_{\pi v}}{2 N}\right) f(m, n)
$$


where $u$ or $v=a$ :

$$
\begin{gathered}
C(a)=2^{-1 / 2} \text { si } a=0 \\
C(a)=1 \text { if }: a=1,2, \ldots, N-1
\end{gathered}
$$

The corresponding inverse DCT transform of each block is:

$$
f(m, n)=\sum_{u=0} \sum_{v=0}^{N-1 N-1} c(u) c(v) F(u, v) \cos \left(\frac{(2 m+1) \pi u}{2 N}\right) \cos \left(\frac{(2 n+1) \pi v}{2 N}\right)
$$

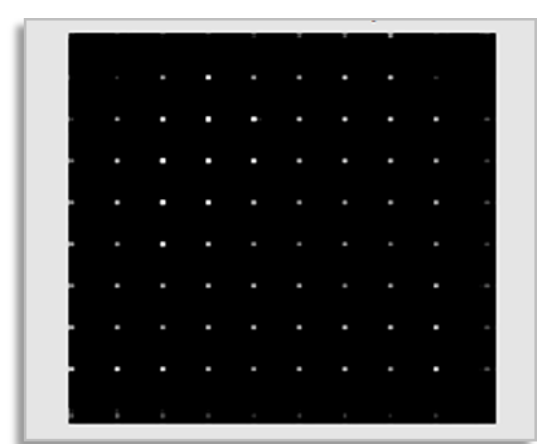

Fig. 4. DCT transform and scalar quantification of test approximation sub-band.

BLOCK A: THE LOW SUB-BAND (WAVELET COEFFICIENTS)

$\left(\begin{array}{lllllllll}112.8457 & 94.7229 & 78.6852 & 99.7675 & 85.2297 & 75.1239 & 95.0060 & 78.8680 \\ 109.0257 & 85.6655 & 92.6332 & 91.0384 & 81.4676 & 74.4327 & 66.5158 & 68.4359 \\ 77.8930 & 82.7769 & 91.0010 & 101.1569 & 76.1003 & 69.8532 & 67.8609 & 66.5018 \\ 77.3548 & 80.3425 & 112.5705 & 118.7605 & 70.9124 & 68.0798 & 63.7924 & 62.8930 \\ 82.1374 & 86.9829 & 181.5624 & 110.8953 & 71.2011 & 66.5830 & 62.7316 & 64.1826 \\ 71.8390 & 192.4753 & 218.5648 & 112.4335 & 72.7729 & 67.4281 & 62.7778 & 64.5674 \\ 106.3542 & 244.0490 & 169.0009 & 112.7637 & 79.0329 & 67.2682 & 64.6210 & 67.7837 \\ 123.4306 & 208.4687 & 177.4095 & 121.3289 & 133.7858 & 72.5128 & 72.1284 & 76.1604\end{array}\right)$

BLOCK B: THE LOW SUB-BAND AFTER TRANSFORM DCT AND QUANTIZATION

$$
\left(\begin{array}{cccccccc}
48 & 16 & -4 & -6 & -2 & -1 & 0 & 0 \\
-9 & -9 & 1 & 4 & 3 & 1 & 0 & 0 \\
4 & 2 & 2 & 2 & 0 & -1 & -1 & 0 \\
1 & 1 & 0 & -1 & -1 & 0 & 0 & 0 \\
0 & -1 & 0 & 0 & 0 & 0 & 0 & 0 \\
0 & 0 & 0 & 0 & 0 & 0 & 0 & 0 \\
0 & 0 & 0 & 0 & 0 & 0 & 0 & 0 \\
0 & 0 & 0 & 0 & 0 & 0 & 0 & 0
\end{array}\right)
$$

Then, comes the step of scalar quantization, which consists of dividing the DCT coefficients by a quantization table in accordance with the desired compression, in which the loss of information occurs. Each block, is reorganized by ordering the coefficients, so that the frequency variations are conducted in a zigzag sequence. This approach allows us to favor low frequencies (refer to [7] for more details). As a final step, we proceed to a RLE coding.

\section{Numerical Example}

Examples of a block extracted from an approximation sub-band, and of one obtained after DCT and scalar quantization, are given in Block A and Block B.

\section{Vector Quantization of Details Sub-bands Using Competitive Learning}

Vector quantization is a powerfull technique and particularly effective for data compression [8], [9]. It is defined as representing any vector $\mathrm{x}$ of dimension $K$ by a vector of the same size, belonging to a finite set called "code-book". The original image is divided into $N$ dimensional vectors; the vectors are blocks of pixel values. Each block of size $K$ is compared with the set of blocks of the codebook. These predefined blocks are called "code-words" or "reproduction vectors".

Then, a comparison is effected, it entails calculating a distance measurement between the block of the original image (vector) and the code-words. The coding is ignorant of the original block and keeps only the index (address) of the near codeword.

The general distance $\mathrm{d}$ applied is the quadratic Euclidean distance, which is equivalent to the minimization of the mean square error:

$$
d(x, y i)=\|x-y i\|^{2}
$$

where $x$ is the original vector and $y$ is the reproduced vector. The decoder simply shows the corresponding code words indices (received or stored), and thus, reconstructs the image.

Wavelet decomposition of an image allows developing a codebook for each resolution level and preferred direction $(H, V, D)$. Each codebook is created from training sequence consisting of vectors that belong to different sub-bands, corresponding to the level of resolution and the direction considered. So, a general codebook may be obtained by assembling these codebooks. Each codebook is low distortion and contains a few words, the result is a clear gain in finding the best vector coding.

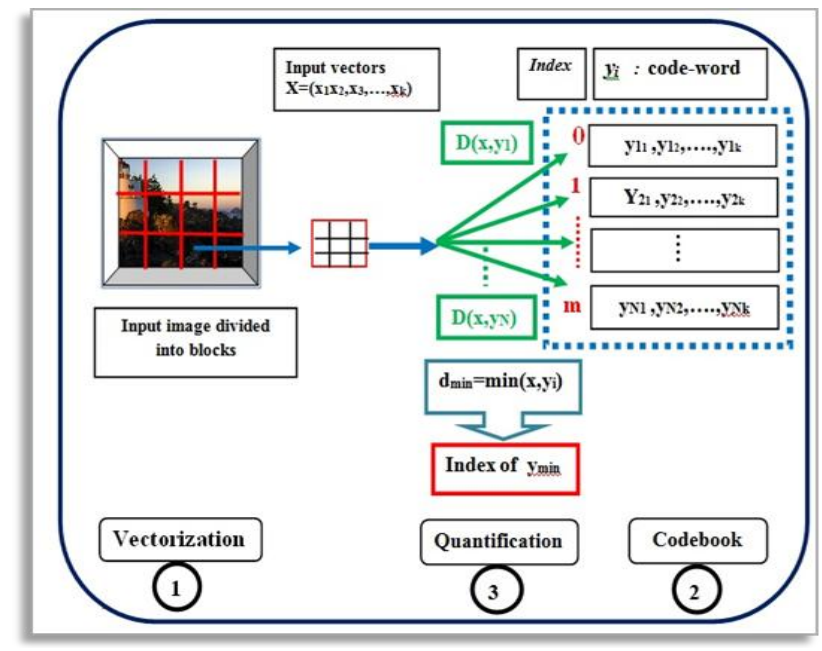

Fig. 5. Processes of quantification.

For generating codebook there exists different algorithms, like Lind, Buzo and Gray (LBG) algorithm [10], Self Organizing Feature Map (SOFM) [11], In the proposed method we used an approach using neural network learning (FSCL: competitive learning sensitive to the frequency), the principle of which is described below. 


\section{RESUlts OBTAINED}

The results are shown in Fig. 8 and Fig. 10. These respectively result from the compression and decompression of visible, infrared Meteosat images of Fig. 6 and Fig. 8. The image compression is carried out with a compression factor of 27.97 , that is to say, a total compression ratio of $96.42 \%$. On these images, no artifact appears. It has reduced the volume of data while maintaining their initials properties.

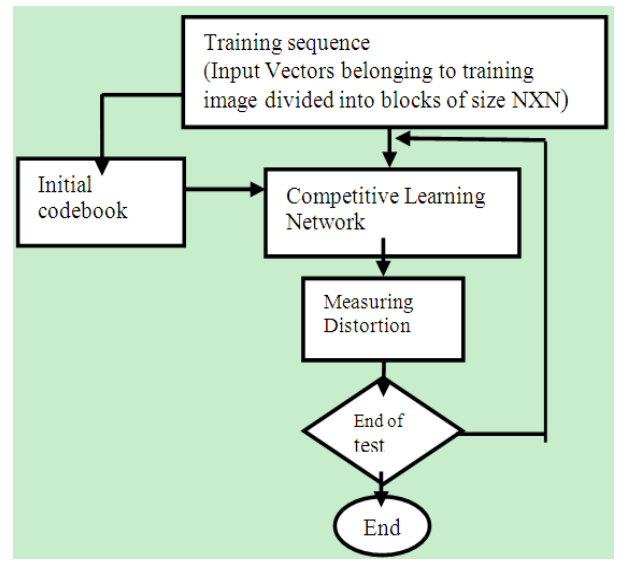

Fig. 6. Schematic FSCL algorithm.

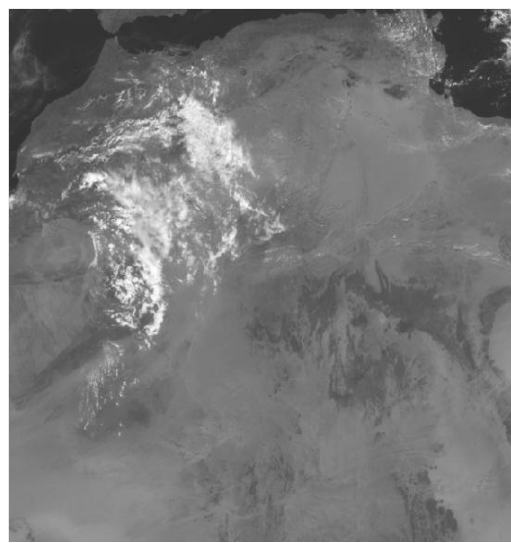

Fig. 7. Original visible image of Meteosat sensor (Size: 600×600pixels).

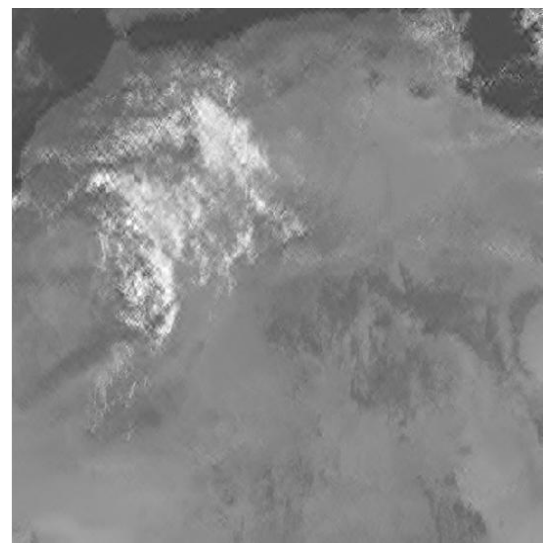

Fig. 8. Reconstructed visible image, with $\mathrm{bpp}=0.2 \mathrm{bits} / \mathrm{p}, \mathrm{PNSR}=30 \mathrm{~dB}$.

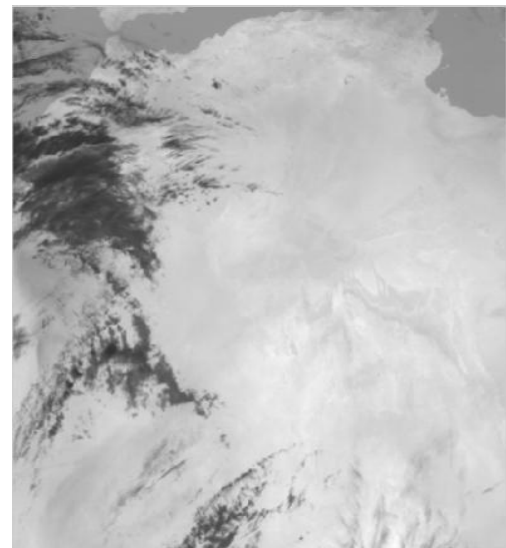

Fig. 9. Original infrared image of Meteosat sensor (size: $600 \times 600$ pixels).

\section{EVALUATION OF EXPERIMENTAL RESULTS AND DISCUSSIONS}

To evaluate the performance of the compression method tested, we carried out two analyses. The first is qualitative analysis of the results dealing with the discussion of the loss of visual quality that occurred during the compression step (visual quality).

A second analysis is of statistical indicators (correlation) and a quantitative one (computing the signal to noise ratio) of the distortion of the compressed information.

\section{A. Results of Qualitative Analysis}

The results show a very good reconstruction of the raw images, the images show difference that coding errors are located primarily on the outlines of the images.This is because the edges are not well represented in the dictionaries. Such errors are imperceptible to the human eye. This is one of the advantages provided by the use of wavelet decomposition.

Where should resolution (3) be used to compress the approximation sub-band because: the initial size of the image being: $600 \times 600 \times 8$ bits, its final size resolution (3) will be: $75 \times 75 \times 8$ bits. The resultant compression factor will be equal to 64 . In so doing, we find that the total rate of compression is significantly reduced and is approximately $89.32 \%$. Also,

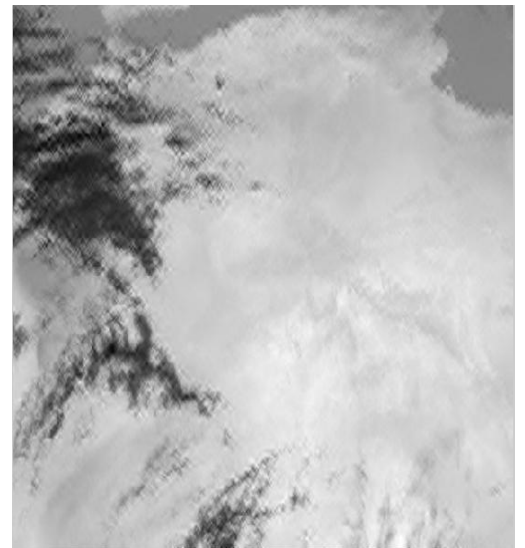

Fig. 10. Reconstructed infrared image, with $b p p=0.2 b i t s / p, P N S R=30 \mathrm{~dB}$

given that this approach ignores the information at high frequencies, we obtain a poor picture quality when uncompressing.

To overcome these drawbacks, the discrete cosine transform, quantization and coding have been introduced in the process of compressing the image. Thus, the application of these factors to the approximation sub-band has allowed us to get good quality, with a better compression ratio of $96.2994 \%$.

\section{B. Results of Quantitative Analysis}

The analysis of the results of the compression refers to the measurement of several statistical indicators. The indicators used in this analysis are the correlation checked graphically and the criterion measure of Peak Signal Noise Ratio (PSNR in $\mathrm{dB}$ ), widely used to assess the quality of digital compression, defined as (Table I and Table II):

$$
P S N R=10 \log _{10} \frac{255^{2}}{\frac{1}{W H} \sum_{i=1}^{W} \sum_{j=1}^{H}\left(X_{i j}-y_{i j}\right)^{2}}
$$

where:

255: the peak gray level of the image,

$x_{i j}, y_{i j}$ : the pixel gray levels from the original and reconstructed images, respectively, 
$W \times H$ : is the total number of pixels in the image.

TABLE I: PEAK SIGNAL NOISE RATIO (PNSR)

\begin{tabular}{llc}
\hline \hline Image tests & PSNR $(\mathrm{dB})$ & Compression ratio \\
\hline Image 1 & 31.75 & 27.16 \\
Image 2 & 35.13 & 30.42 \\
Image 3 & 37.67 & 34.13 \\
\hline \hline
\end{tabular}

TABLE II: COMPARISON OF THE PROPOSED METHOD WITH JPEG METHOD

\begin{tabular}{lllll}
\hline \hline & $\begin{array}{l}\text { Same } \\
\text { compression } \\
\text { factor }\end{array}$ & $\begin{array}{l}\text { Time of } \\
\text { compression }\end{array}$ & $\begin{array}{l}\text { Time of } \\
\text { decompression }\end{array}$ & $\begin{array}{c}\text { Performance } \\
\text { PSNR (dB) }\end{array}$ \\
\hline $\begin{array}{l}\text { Visible: } \\
\text { JPEG Our }\end{array}$ & $37: 1$ & 15.54 & $1 \mathrm{~s}$ & 36.46 \\
result & $37: 1$ & 34.762 & $1.055 \mathrm{~s}$ & 40.10 \\
\hline $\begin{array}{l}\text { Infrared: } \\
\text { JPEG }\end{array}$ & $37: 1$ & 12.036 & $1 \mathrm{~s}$ & 27.34 \\
$\begin{array}{c}\text { Our } \\
\text { result }\end{array}$ & $37: 1$ & 32.023 & $1.053 \mathrm{~s}$ & 31.83 \\
\hline \hline
\end{tabular}

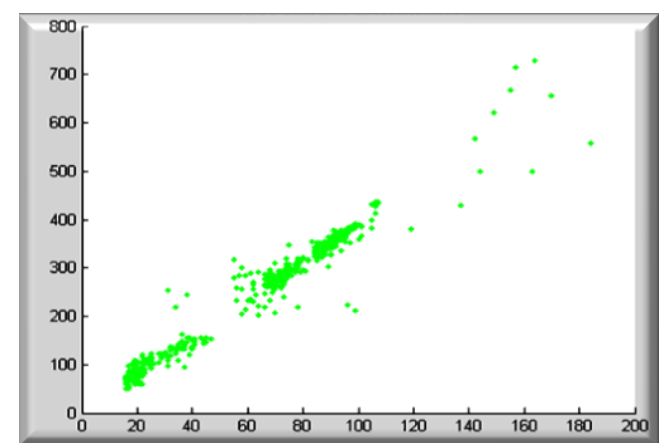

Fig. 11. Scatter plot of correlation between original visible image and reconstructed image.

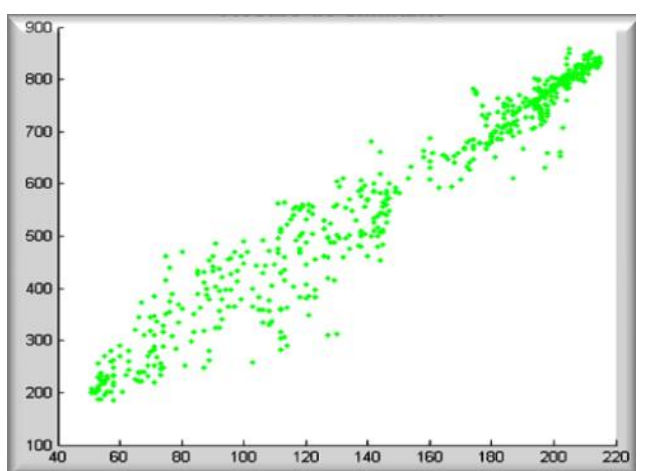

Fig. 12. Scatter plot of correlation between original infrared image and reconstructed image.

The statistical method (statistical correlation) observed between the original image and the compressed image is a measure of precision of the result of compression that can be verified graphically with scatter plots, and numerically by calculating the correlation coefficient. In our case, we will use the graphical method "scatter plots". The scatter plots can be obtained by plotting the points $\left(x_{i}, y_{i}\right)$ where $x_{i}$ is the value pixel at position from image $X$, and $y_{i}$ is the value at the same pixel position from image $Y$.

If the images are correlated, then these points will cluster around the 45-degree line passing through the origin [12].
The Fig. 11 and Fig. 12 illustrate the scatter plot resulting from comparing the values of visible and infrared images:

\section{CONCLUSION}

This work is dedicated to the exploration of a new compression method for digital images, introducing a double process of compression: lossless compression (the scalar quantization) to preserve the quality of the image, and, with compression containing losses (vector quantization) to improve the compression ratio, with a slight degradation in quality. The experimental results show, that the double process of quantification can greatly reduce the volume ofdata, with a good visual quality (images are reproduced faithfully), while preserving their original properties. But, the compression of an image requires time for calculation. As a prospect for future research, this method can be easily extended to color images by processing the three color matrices separately.

\section{REFERENCES}

[1] Y. lano, D. S. Silvestre, and A. L. M. Cruz, "A fast and efficient hybrid fractal-wavelet image coder," IEEE Trans. Image Process, vol. 15, no. 1, pp. 98-105, 2006

[2] F. Borko, "A survey of multimedia compression techniques and standards," Part I JPEG standard, Real-Time Imaging, no. 1, pp. 49-67, 1995

[3] G. Held and R. D. Marshall, Data and Image Compression tools and Techniques, John Wiley \& Sons, Chichester, 1996, p. 432.

[4] T.-C. Lu and C.-Y. Chang, "A survey of VQ codebook generation," Ubiquitous International Journal of Information Hiding and Multimedia Signal Processing, vol. 1, no. 3, July 2010.

[5] S. Radhakrishnan and J. Subramaniam, "Novel image compression using multi-wavelets with SPECK algorithm," International Arab Journal of Information Technology, vol. 5, no. 1, pp. 45-51, 2008.

[6] K. R. Rao and P. Yip, "Discrete cosine transform: Algorithms, advantages, applications," New York: Academic, 1990.

[7] S. V. Viraktamath and V. Attimarad, "Impact of quantization matrix on the performance of JPEG," International Journal of Future Generation Communication and Networking, vol. 4, no. 3, pp. 107-118, September 2011.

[8] A. Gersho, "One the structure of vector quantizers," IEEE Trans. on Inform Theory, vol. IT-28, no. 2, 1982.

[9] A. Gersho and R. M. Gray, "Vector quantization and signal compression," Springer International Series in Engineering and Computer Science, vol. 159, 1992, ch. 10, pp. 309-343.

[10] Y. Linde, A. Buzo, and R. M. Gray, "An algorithm for vector quantizer design," IEEE Trans. Communications, vol. 28, no. 1, pp. 84-95, January 1980.

[11] T. Kohonen, Self Organizing Maps, 3rd ed. Springer, 2001.

[12] R. Ashraf, "Diagnostically lossless compression of medical images," $\mathrm{Ph}$.D. Thesis, Dept. Elect. Eng, Miltary College of signals, Rawalpinidi University of sciences and technology, Pakistan, June 2008.

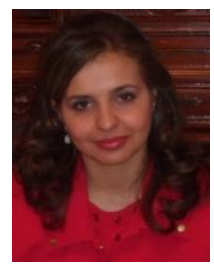

Amel Bey Boumezrag received her ING. degree in electronics and communication engineering from the University of Laghouat, and her master of science in communication systems \& networks, in 2012. Currently, she is training as an engineer of information technology and communication in telecom. Her field of interest includes signal and image processing, transmission, communication systems and networks, remote sensing. 